\title{
Endoscopic ultrasound fine-needle aspiration by experienced pulmonologists: a cusum analysis
}

\author{
Paul Leong ${ }^{1,2,3}$, Sheetal Deshpande ${ }^{1}$, Louis B. Irving ${ }^{3}$, Philip G. Bardin ${ }^{1,2}$, \\ Michael W. Farmer ${ }^{1,2}$, Barton R. Jennings ${ }^{1}$ and Daniel P. Steinfort ${ }^{3,4}$ \\ Affiliations: ${ }^{1}$ Monash Lung and Sleep, Monash Health, Clayton, Australia. ${ }^{2}$ Faculty of Medicine, Nursing and \\ Health Sciences, Monash University, Clayton, Australia. ${ }^{3}$ Dept of Respiratory Medicine, Melbourne Health, \\ Parkville, Australia. ${ }^{4}$ Dept of Medicine, University of Melbourne, Melbourne, Australia.
}

Correspondence: Paul Leong, c/o Monash Lung and Sleep, 246 Clayton Rd, Clayton, Victoria 3168, Australia. E-mail: paul.leongamonashhealth.org

@ERSpublications

Endoscopic ultrasound fine-needle aspiration can be performed with an endobronchial scope http://ow.ly/LtPd30fwkn1

Cite this article as: Leong P, Deshpande S, Irving LB, et al. Endoscopic ultrasound fine-needle aspiration by experienced pulmonologists: a cusum analysis. Eur Respir J 2017; 50: 1701102 [https://doi.org/10.1183/ 13993003.01102-2017].

ABSTRACT Endobronchial ultrasound transbronchial needle aspiration (EBUS TBNA) is an established, minimally invasive way to sample intrathoracic abnormalities. The EBUS scope can be passed into the oesophagus to perform endoscopic ultrasound with bronchoscope-guided fine-needle aspiration (EUS-BFNA). In cases of suspected lung cancer, a combination of the two techniques is now recommended by consensus guidelines. EBUS TBNA is usually performed by pulmonologists; however, the learning curve for EUS-B-FNA, which may be performed during the same procedure, has not been described.

A multicentre, observational Australian study, using prospectively collected data from three experienced pulmonologists was conducted. Cumulative sum (cusum) analysis was used to generate visual learning curves.

A total of 152 target lesions were sampled in 137 patients, with an overall sensitivity for malignancy of 94.8\%. The sensitivity for malignant lesions outside of the 2009 International Association for the Study of Lung Cancer lymph node map (largely intraparenchymal lesions) was 92.9\%. All three operators were competent by conventional cusum criteria. There was one case of pneumothorax, and no episodes of mediastinitis or oesophageal perforation were observed.

Our data suggest that experienced pulmonologists can safely and accurately perform EUS-B-FNA, with a high diagnostic sensitivity for both lymph node and non-nodal lesions. 


\section{Introduction}

The advent of the linear array endobronchial ultrasound bronchoscope (EBUS) has facilitated the minimally invasive sampling of structures that are adjacent to the airways, in a technique known as EBUS transbronchial needle aspiration (TBNA). This technique allows sampling of paratracheal, subcarinal and hilar nodal stations [1].

The curvilinear endobronchial bronchoscope can also be passed into the oesophagus and the stomach, thereby allowing adjacent structures to be sampled, using endoscopic ultrasound with bronchoscope-guided fine-needle aspiration (EUS-B-FNA) [2]. This technique increases the sensitivity of EBUS TBNA to detect mediastinal lymph node metastases, and might improve bronchoscopic yield in the investigation of peripheral pulmonary lesions [3].

EBUS TBNA and EUS-B-FNA are complementary techniques for the diagnosis of lung cancer, and the 2015 Expert Consensus guidelines suggest the combination of the two techniques is preferred over either alone [4]. The learning curve for EBUS TBNA has been examined [5-7].

Whilst the adoption of EBUS TBNA has grown substantially over the past 5 years, with established recommendations for training [8], EUS-B-FNA is performed relatively infrequently, and no guidelines presently exist regarding the recommended training required to establish competency. To our knowledge, only one study has examined the learning curve for EUS in pulmonologists, which focused on the use of the gastrointestinal echoendoscope by bronchoscopists who had no experience in EBUS TBNA, and reported variable performance, with only two out of four participants attaining an expert standard [9].

Pulmonologists familiar with EBUS TBNA have skills in interpretation of linear EBUS imaging, as well as handling of the curvilinear videobronchoscope, and we hypothesised that such proceduralists would demonstrate more rapid attainment of competence in EUS-B than has been previously reported for bronchoscopists undertaking EUS. We set out to describe the learning curves for EUS-B-FNA, to determine whether experienced EBUS TBNA operators could safely and adequately perform EUS-B-FNA.

\section{Methods}

The learning curves of three operators (M.W. Farmer, B.R. Jennings and D.P. Steinfort) are described in the present analysis. All three are board certified pulmonologists. Procedures were performed at four major tertiary teaching centres in Melbourne, Australia that employed selective targeting of lesions to achieve staging or pathologic diagnosis in the most efficient fashion, generally starting with the lesion that would yield the highest stage, if malignant. Rapid onsite cytology by an experienced cytologist was available during all procedures. All operators had EBUS TBNA experience prior to the commencement of EUS-B-FNA, with a minimum 150 EBUS TBNA procedures each. We have previously described the teaching/training in EUS-B undertaken at the beginning of this cohort study [2]. None of the operators had prior EUS-B-FNA experience or training, nor had they performed any gastrointestinal endoscopy.

Procedures were performed under moderate to deep sedation by board certified anaesthesiologists, as previously described [10]. One operator always employed a laryngeal mask airway, whereas the other two operators employed no airway aid, other than an oral bite block. In general, the sedation provided was the same as would be administered for EBUS TBNA. When both EBUS TBNA and EUS-B-FNA were indicated, EBUS TBNA was performed first.

Cusum (cumulative sum) analysis [11] was used to produce a learning curve for each bronchoscopist. In brief, cusum is a graphical method of depicting learning and the achievement of predefined competency or non-competency. Cusum offers a technique that can be readily applied to not only to learners, but also to practising clinicians, and provide a stimulus for retraining or even proof of competency for credentialing. On a cusum plot, cases are represented along the horizontal axis, and the outcome, along the vertical axis. The proceduralist begins at the intersection of the axes, and each case adds or subtracts to the proceduralist's 'cumulative sum'. Positive deflections indicate failures, and negative deflections indicate successful procedures. The penalty for a failure exceeds the reward for a success. Using Kestin's method [12], acceptable performance is denoted by a cusum progression that is horizontal or by downsloping.

Boundary lines plotted parallel to the $\mathrm{x}$-axis that represent predefined acceptable performance thresholds at a given number of cases, incorporate Type I and II error rates, as well as acceptable and unacceptable failure rates. Crossing a boundary from above is an indicator of a successful procedural record, and crossing a boundary from below is an indicator of an unsuccessful procedural record. Cusum is responsive to interventions, including retraining, and has been used to analyse learning in a variety of procedures and specialties including EBUS TBNA [11].

For the present study, the following definitions were adapted from Kemp et al. (2010) [5]. 
True positive: 1) EUS-B-FNA cytology diagnostic of malignancy, or other process; 2) atypical and suspicious cytopathologic diagnoses confirmed on later tissue samples.

True negative: 1) EUS-B-FNA cytology and subsequent investigations confirmed non-cancer diagnosis; 2) EUS-B-FNA cytology negative or inconclusive, with subsequent investigations, and patient alive and well at 6 months, with original abnormalities clinically ascribed to a non-cancer diagnosis; 3) EUS-B-FNA cytology negative or inconclusive and subsequent investigations confirmed non-malignant involvement of sampled region.

False negative: 1) EUS-B-FNA cytology negative, but subsequent investigations confirmed a diagnosis of cancer; 2) EUS-B-FNA cytology negative with inconclusive or no subsequent investigations and progressive disease clinically ascribed to cancer; 3) EUS-B-FNA cytology negative with inconclusive or no subsequent investigations, but patient not alive at 6 months, with death ascribed to related cancer; 4) EUS-B-FNA cytology negative with inconclusive or no subsequent investigations, and patient lost to follow-up; 5) technical failure, unsatisfactory specimen.

False positive: EUS-B-FNA cytology positive for malignancy, but histopathology at biopsy/resection/ post-mortem showed different malignancy.

Consistent with the procedures of KeMP et al. [5], we set the Type I and II error criteria at 10\%, with the acceptable failure rate set at $10 \%$ and unacceptable failure rate at $20 \%$. A priori, we did not restrict our analysis to mediastinal lymphadenopathy.

\section{Results}

A review of prospectively maintained databases by the proceduralists and two independent authors (P. Leong and S. Deshpande) identified 152 target lesions, sampled in 137 patients. Features of the lesions sampled are detailed in tables 1 and 2. With regards to malignancy, there were 92 true positive cases, 40 true negatives, zero false positives and five false negatives (table 2, details of false negative cases are shown in table 3). Overall, this represents a $94.8 \%$ sensitivity for malignancy. In our cohort, the malignancy prevalence was high at $70.8 \%$.

Among the target lesions, 42 (27.6\%) occurred in locations outside the International Association for the Study of Lung Cancer (IASLC) 2009 lymph node map [13], of which the majority were intraparenchymal masses (81.0\%, 34 out of 42) (table 1). For non-IASLC targets, sensitivity for definite malignancy was 92.9\% (39 out

\section{TABLE 1 Endoscopic ultrasound with bronchoscope-guided fine-needle aspiration targets}

\begin{tabular}{|c|c|c|c|}
\hline \multirow[t]{2}{*}{ Anatomical location ${ }^{\#}$} & \multicolumn{3}{|c|}{ Target lesions sampled $\mathbf{n}$} \\
\hline & $\begin{array}{l}\text { Operator } 1^{\text {ก }} \text { over } \\
4.5 \text { years }\end{array}$ & $\begin{array}{c}\text { Operator } 2^{+} \text {over } \\
3.5 \text { years }\end{array}$ & $\begin{array}{c}\text { Operator } 3^{\S} \text { over } \\
1.5 \text { years }\end{array}$ \\
\hline \multicolumn{4}{|l|}{ Nodal station } \\
\hline $1 \mathrm{~L}$ & 0 & 1 & 0 \\
\hline $2 \mathrm{~L}$ & 4 & 0 & 2 \\
\hline $2 \mathrm{R}$ & 7 & 1 & 0 \\
\hline $3 p$ & 2 & 1 & 1 \\
\hline $4 \mathrm{~L}$ & 10 & 9 & 10 \\
\hline 5 & 0 & 1 & 5 \\
\hline 7 & 9 & 20 & 9 \\
\hline 8 & 1 & 9 & 3 \\
\hline 9 & 1 & 0 & 1 \\
\hline $11 \mathrm{~L}$ & 0 & 0 & 1 \\
\hline \multicolumn{4}{|l|}{ Non-nodal lesions } \\
\hline Right upper lobe & 4 & 2 & 7 \\
\hline Right lower lobe & 0 & 4 & 2 \\
\hline Left upper lobe & 3 & 7 & 5 \\
\hline $\begin{array}{l}\text { Low paraoesophageal } \\
\text { lesions }\end{array}$ & 3 & 1 & 0 \\
\hline Coeliac axis node & 0 & 1 & 0 \\
\hline Left adrenal gland & 0 & 2 & 0 \\
\hline Pleura & 0 & 1 & 0 \\
\hline
\end{tabular}

\footnotetext{
\#: lymph node stations are listed as per the International Association for the Study of Lung Cancer 2009
} classification [13]; ๆ: 43 patients, 44 targets; $^{+}$: 50 patients, 60 targets; ${ }^{\S}$ : 44 patients, 46 targets. 
TABLE 2 Procedural diagnosis by endoscopic ultrasound with bronchoscope-guided fine-needle aspiration and diagnostic performance

\begin{tabular}{|c|c|c|c|}
\hline Category & Operator 1 & Operator 2 & Operator 3 \\
\hline \multicolumn{4}{|l|}{ Diagnosis } \\
\hline Nonsmall cell lung cancer & 19 & 20 & 15 \\
\hline Small cell lung cancer & 7 & 3 & 3 \\
\hline Other solid organ malignancy & 3 & 6 & 5 \\
\hline Lymphoma & 3 & 3 & 1 \\
\hline Sarcoidosis & 4 & 7 & 9 \\
\hline Mesothelioma & 0 & 1 & 0 \\
\hline Benign & 5 & 8 & 9 \\
\hline Atypical cells & 2 & 1 & 2 \\
\hline \multicolumn{4}{|l|}{ Diagnostic performance } \\
\hline True positives & 32 & 34 & 26 \\
\hline True negatives & 9 & 14 & 17 \\
\hline False positives & 0 & 0 & 0 \\
\hline False negatives & 2 & 2 & 1 \\
\hline Sensitivity $\%$ & 94.1 & 94.4 & 96.3 \\
\hline Specificity \% & 100 & 100 & 100 \\
\hline Complications & $\begin{array}{c}\text { Atrial fibrillation } 1, \\
\text { Exacerbation of COPD } 1\end{array}$ & Pneumothorax 1 & Nil \\
\hline
\end{tabular}

Data are presented as $n$, unless otherwise stated.

of 42). Of the remaining three lesions, two were truly negative (one cyst and one abscess), and one was presumed false negative (a PET-avid left adrenal gland was sampled; however, a left upper lobe lung mass sampled during the same procedure demonstrated nonsmall cell lung cancer (NSCLC)).

For IASLC nodal lesions, the sensitivity was $89.0 \%$, and the negative predictive value was $84.0 \%$.

Cusum curves for all three proceduralists were downsloping and were consistent with acceptable performance (figure 1).

\section{Complications}

No episodes of mediastinitis or oesophageal perforation occurred. One pneumothorax $(0.7 \%$, $95 \%$ CI $0.1 \%-4 \%$ ) occurred in a patient with an intraparenchymal right upper lobe mass, $1 \mathrm{~cm}$ from the oesophagus following five samplings of the lesion. The procedure yielded a diagnosis of adenocarcinoma, and the pneumothorax was managed with an intercostal catheter that was removed after 2 days. One patient had an acute exacerbation of chronic obstructive pulmonary disease (COPD), and another experienced new post procedure atrial fibrillation without haemodynamic instability.

\section{Discussion}

In this study, we depict the cusum learning curves of three experienced EBUS TBNA operators, as they began to perform EUS-B-FNA in a study population with a high malignancy prevalence. Despite

\section{TABLE 3 Characteristics of false-negative cases}

\section{False-negative status Comment}

\section{Presumed}

Confirmed

Presumed

Confirmed

Confirmed
2R (22-mm long axis) sampled after EBUS TBNA 4R (13 mm), 4 L (13 mm), all samples reported as "atypical cells". All nodes were PET ${ }^{\Uparrow}$-avid. Metastatic carcinoma (no subtype possible) diagnosed from fine-needle aspirate of PET-avid right supraclavicular node $(15 \mathrm{~mm})$.

PET-avid 2R $(20 \mathrm{~mm})$ sampled; sample contained lymphocytes, lymphoma eventually diagnosed on mediastinoscopy. Station 7 sampled, lymphocytes only, lost to follow-up, so assigned false negative.

PET-avid stations $7(25 \mathrm{~mm})$ and $2 \mathrm{R}(18 \mathrm{~mm})$ sampled for mediastinal lymphadenopathy in the context of prior colorectal cancer. Non-necrotising granulomatous inflammation on cytology, Hodgkin's lymphoma diagnosed on cervical lymph node excisional biopsy $(20 \mathrm{~mm})$.

Station 7 sampled and lymphocytes only reported; resection demonstrated a $0.5 \mathrm{~mm}$ focus of metastatic disease. 
a)

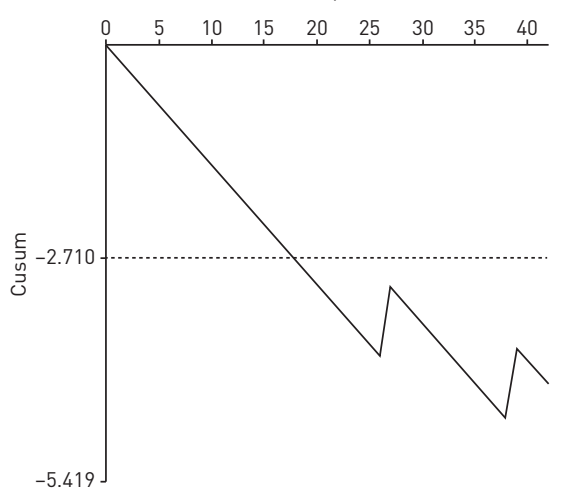

b)

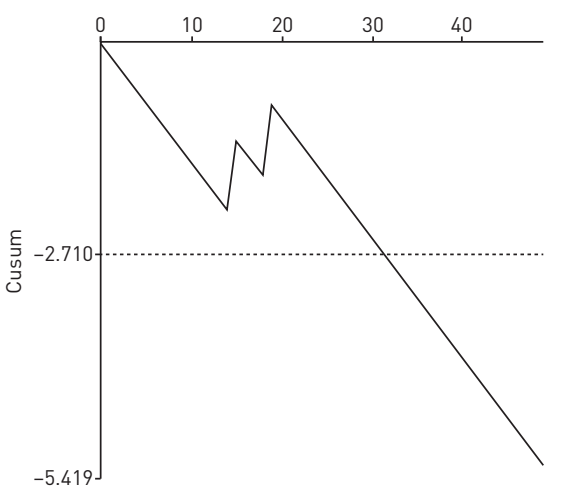

c)

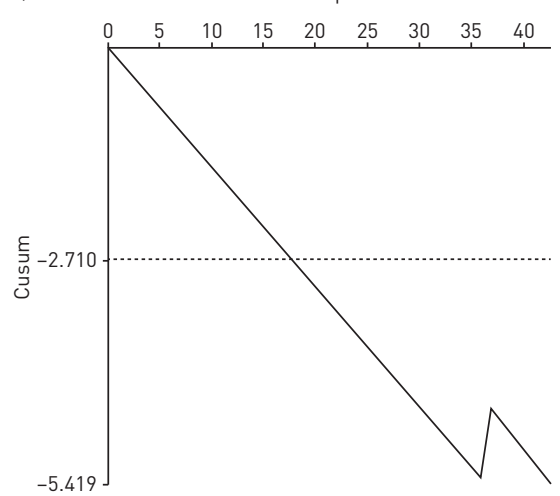

FIGURE 1 Cumulative sum (cusum) plots for three operators performing endoscopic ultrasound with bronchoscope-guided fine-needle aspiration. In cusum analysis, the operator starts at the origin of the axes, with the number of cases indicated on the horizontal axis and "performance" indicated on the vertical axis. Each case provides a downward (true-positive or true-negative) or upward (false-positive or false-negative) data point. Downward-sloping plots, which cross predefined horizontal lines from above are indicative of competence. All three operators appear competent.

conservative criteria for acceptable/unacceptable failure rates that were considered 'tough' by KEMP et al. [5, 6], all three operators appeared immediately competent, with roughly equal numbers of cases. Importantly, we observed a very low rate of complications.

Proceduralists demonstrated performance that was largely consistent among the three. Our experience indicates that it is possible for EUS-B-FNA to be performed by pulmonologists who are experienced in EBUS TBNA, with favourable diagnostic performance and safety. This appears to apply to both EUS-B-FNA of lymph node lesions, as well as parenchymal lung lesions. Two of the eight false negatives occurred in lymphoma, a condition in which the EBUS TBNA yield is suboptimal [14]. It is noteworthy that timely surgical biopsy was arranged for these cases when the specimens were returned as non-diagnostic, highlighting the importance of clinical suspicion of lymphoma, and an awareness of the diagnostic limitations of this technique.

The diagnostic sensitivity of EUS-B-FNA for non-IASLC lesions in this study was $92.9 \%$, driven largely by intraparenchymal lesions, and comparable to the yield of $90 \%$ and sensitivity of $92 \%$ for intraparenchymal lesions in a recent meta-analysis [15]. Most patients in our cohort underwent EUS-B-FNA for diagnostic purposes, with only a minority of procedures performed for mediastinal lymph node staging of NSCLC. Thus, we are unable to examine the additional improvement in ' $N$ ' staging of NSCLC, following the addition of EUS-B-FNA to EBUS TBNA. However, numerous carefully designed prospective trials have already addressed this question, and confirmed that EUS-B-FNA enhances the accuracy of staging beyond that of EBUS TBNA alone [16, 17]. Meta-analyses have identified the number of combined endosonographic procedures (EUS-B-FNA added to EBUS TBNA) that need to be performed to identify one patient with occult N2/3 involvement by NSCLC to be $10-14[18,19]$. It is therefore not surprising that a combination of the two procedures has now been recommended by a recent European guideline [4]. The adoption of EUS-B-FNA is likely to be increasingly important with greater use of systematic lymph node staging prior to radical [20] or stereotactic treatment of NSCLC [21].

Cusum has caveats. The adjudication of cases is dependent on the definitions used, and in order to err on the side of caution, cases with inadequate follow-up or unclear diagnoses at death are assigned as false negative. This might have the effect of falsely accusing the proceduralist of incompetence. An additional effect is that as operators gain more experience, they often intentionally accept difficult cases, in which the probability of success is low to moderate, in an attempt to spare more invasive procedures. It is reassuring that despite these factors, all three proceduralists examined by cusum in the present study, demonstrated competence within these constraints.

As linear array EBUS becomes increasingly available and confidence grows with EBUS TBNA, it is likely that more operators will be tempted to perform EUS-B-FNA, given that the same instrument could be used for both procedures in the same setting. We would recommend close and ongoing evaluation of diagnostic and safety outcomes for all proceduralists commencing EUS-B-FNA.

Proceduralists commencing EUS-B-FNA should be aware of unique anatomical considerations of this procedure and its potential complication profile, and should consider having the supervision of a colleague who is experienced in gastrointestinal endoscopy. It is worth noting that all proceduralists in the present 
study were highly experienced in the performance of EBUS TBNA. Cusum curves are likely to differ in proceduralists who are less experienced with EBUS TBNA. Validated tools such as the EUS Assessment Tool (EUSAT) [22] could aid learning, including anatomical recognition and foster procedural fluency. As in EBUS TBNA, the development and introduction of quality standards [23, 24] and structured society training programmes [25], could reduce practice variation and increase quality.

The EUS-B-FNA technique can be considered as more difficult than EBUS TBNA, given that there are few visual cues or landmarks on the luminal view; however, during sampling, cartilage rings do not present access problems. Respiratory movement is more pronounced on EUS-B-FNA than on EBUS TBNA, and oesophageal sliding can make targeting difficult on occasion. Despite these challenges, we demonstrated good diagnostic performance and safety. We suggest that familiarity with the technique of EBUS TBNA puncture and its limitations might have accelerated the learning curve for EUS-B-FNA.

\section{Conclusions}

Our study indicates that experienced pulmonologists are able to safely and accurately perform EUS-B-FNA, with a high diagnostic sensitivity for both lymph node and non-nodal lesions.

Further research is required to determine whether competence in EUS-B-FNA parallels that of EBUS TBNA, and whether theoretical training, simulations or other workshop-based training aids could hasten the learning curve.

\section{Acknowledgements}

We thank the endoscopy staff at our respective sites.

\section{References}

1 Tournoy KG, Annema JT, Krasnik M, et al. Endoscopic and endobronchial ultrasonography according to the proposed lymph node map definition in the seventh edition of the tumor, node, metastasis classification for lung cancer. J Thorac Oncol 2009; 4: 1576-1584.

2 Wimaleswaran H, Farmer MW, Irving LB, et al. Pulmonologist-performed transoesophageal sampling for lung cancer staging using an endobronchial ultrasound video-bronchoscope: an Australian experience. Intern Med J 2017; 47: 205-210.

3 Steinfort DP, Farmer MW, Irving LB, et al. Pulmonologist-performed per-esophageal needle aspiration of parenchymal lung lesions using an EBUS bronchoscope: diagnostic utility and safety. J Bronchol Interv Pulmonol 2017; 24: 117-124.

4 Vilmann P, Clementsen PF, Colella S, et al. Combined endobronchial and esophageal endosonography for the diagnosis and staging of lung cancer. Endoscopy 2015; 47: 545-559.

5 Kemp SV, El Batrawy SH, Harrison RN, et al. Learning curves for endobronchial ultrasound using cusum analysis. Thorax 2010; 65: 534-538.

6 Medford ARL. Learning Curve for EBUS-TBNA: longer than we may think. Respiration 2015; 90: 173.

7 Steinfort DP, Hew MJ, Irving LB. Bronchoscopic evaluation of the mediastinum using endobronchial ultrasound: a description of the first 216 cases carried out at an Australian tertiary hospital. Intern Med J 2011; 41: 815-824.

8 Wahidi MM, Herth F, Yasufuku K, et al. Technical aspects of endobronchial ultrasound-guided transbronchial needle aspiration: CHEST guideline and expert panel report. Chest 2016; 149: 816-835.

9 Konge L, Annema J, Vilmann P, et al. Transesophageal ultrasonography for lung cancer staging: learning curves of pulmonologists. J Thorac Oncol 2013; 8: 1402-1408.

10 Steinfort DP, Irving LB. Patient satisfaction during endobronchial ultrasound-guided transbronchial needle aspiration performed under conscious sedation. Respir Care 2010; 55: 702-706.

11 Bolsin S, Colson M. The use of the cusum technique in the assessment of trainee competence in new procedures. Int J Qual Health Care 2000; 12: 433-438.

12 Kestin IG. A statistical approach to measuring the competence of anaesthetic trainees at practical procedures. $\mathrm{Br} J$ Anaesth 1995; 75: 805-809.

13 Rusch VW, Asamura H, Watanabe $\mathrm{H}$, et al. The IASLC Lung Cancer Staging Project: a proposal for a new international lymph node map in the forthcoming seventh edition of the TNM Classification for Lung Cancer. $J$ Thorac Oncol 2009; 4: 568-577.

14 Steinfort DP, Conron M, Tsui A, et al. Endobronchial ultrasound-guided transbronchial needle aspiration for the evaluation of suspected lymphoma. $J$ Thorac Oncol 2010; 5: 804-809.

15 Korevaar DA, Colella S, Spijker R, et al. Esophageal endosonography for the diagnosis of intrapulmonary tumors: a systematic review and meta-analysis. Respiration 2017; 93: 126-137.

16 Crombag L, Stigt J, Dooms C, et al. Added value of EUS-B to EBUS for lung cancer staging. Eur Respir J 2015; 46: Suppl. 59, PA772.

17 Sosin $Ł T$, Grochowski Z, Gil T, et al. Minimally invasive strategy for mediastinal staging of patients with lung cancer. Pol Arch Med Wewn 2015; 125: 910-913.

18 Korevaar DA, Crombag LM, Cohen JF, et al. Added value of combined endobronchial and oesophageal endosonography for mediastinal nodal staging in lung cancer: a systematic review and meta-analysis. Lancet Respir Med 2016; 4: 960-968.

19 Dhooria S, Aggarwal AN, Gupta D, et al. Utility and safety of endoscopic ultrasound with bronchoscope-guided fine-needle aspiration in mediastinal lymph node sampling: systematic review and meta-analysis. Respir Care 2015; 60: $1040-1050$. 
20 Steinfort DP, Siva S, Leong TL, et al. Systematic endobronchial ultrasound-guided mediastinal staging versus positron emission tomography for comprehensive mediastinal staging in nsclc before radical radiotherapy of non-small cell lung cancer: a pilot study. Medicine (Baltimore) 2016; 95: e2488.

21 Vial MR, Khan KA, O'Connell O, et al. Endobronchial ultrasound-guided transbronchial needle aspiration in the nodal staging of stereotactic ablative body radiotherapy patients. Ann Thorac Surg 2017; 103: 1600-1605.

22 Konge L, Vilmann P, Clementsen P, et al. Reliable and valid assessment of competence in endoscopic ultrasonography and fine-needle aspiration for mediastinal staging of non-small cell lung cancer. Endoscopy 2012; 44: 928-933.

23 Evison M, Crosbie P, Navani N, et al. How should performance in EBUS mediastinal staging in lung cancer be measured? Br J Cancer 2016; 115: e9.

24 Evison M, Crosbie P, Martin J, et al. EBUS-guided mediastinal lung cancer staging: monitoring of quality standards improves performance. Thorax 2016; 71: 762-763.

25 Farr A, Clementsen P, Herth F, et al. Endobronchial ultrasound: launch of an ERS structured training programme. Breathe 2016; 12: 217-220. 